\title{
About the influence of wheel-rail adhesion on the maximum speed of trains
}

\author{
Marius-Adrian Spiroiu* \\ University POLITEHNICA of Bucharest, Department of Rolling Stock, Splaiul Independenţei 313, \\ Bucharest, Romania
}

\begin{abstract}
Wheel-rail adhesion is a fundamental aspect in rail transport, with high impact on traction and braking of trains. In the case of high speeds trains, wheel-rail adhesion is particularly important, as the maximum usable traction force is limited by the adhesion force. The present paper analyses the influence of wheel-rail adhesion on the maximum speed of trains, considering ranges of usual values for the track gradient and the adhesion coefficient and the real data of two Japanese high-speed trains. Analysis shows that low adhesion and high track gradient result in significant reduction of train speed.
\end{abstract}

\section{Introduction}

The constraints affecting high-speed rail transport are generally related to phenomena of low impact at conventional speeds but which have a high intensity at high speeds. Among these limitations of the vehicles speed can be mentioned: the wheel-rail dynamic forces, the hunting motion, the issues related to current collection, the wheel-rail adhesion, etc [1-3].

Adhesion is a fundamental aspect in rail transport, allowing the transfer of tangential forces between wheel and rail, thus being an essential factor in traction and braking of trains. Low adhesion leads to a limitation of acceleration (during traction process) and of deceleration (in the case of adhesion-based braking). Low adhesion in braking is a safety issue, by the extended stopping distances, while in traction it is a performance issue, in terms of maximum speed and acceleration time - especially when the train is running on steep gradients $[1,4]$.

Low adhesion is caused by the existence of a layer between wheel and rail, for example in cases of water, ice, dead leaves, oil, sand, etc. Since it is the most undesirable case, many studies are investigating the wheel-rail adhesion characteristics in degraded conditions, usually in the case of contaminated surfaces [5-7], with the aim to develop models able to reproduce as accurate as possible the adhesion conditions.

Theoretical and experimental research has shown that adhesion depends on vehicle speed and on wheel-rail creep, which is defined as the ratio between the slip (creep) velocity and the wheelset (vehicle) speed. In the low creep range, the adhesion increases with increased creep until a certain value of creep (1-2\% under dry conditions), when it reaches a maximum. For higher creep, the adhesion decreases as the creep increases. On the

\footnotetext{
${ }^{*}$ Corresponding author: marius_spiroiu@yahoo.com
} 
other hand, regarding the influence of vehicle speed, all analysis carried out in this area has shown that the adhesion is decreasing as the train speed increases.

\section{Wheel-rail adhesion}

Developing an accurate wheel-rail adhesion model exclusively through analytical methods is difficult to achieve, given the complexity of phenomena at the contact surfaces. This is mainly because of the influence of many factors (most of them random) which makes the adhesion to significantly differ from one track section to another, or for the same section, from day to day or even from one hour to the next one. The factors influencing adhesion are various, the most important being the condition of the surfaces in contact (in this regard, atmospheric conditions - temperature, humidity etc. are very important), the vehicles characteristics (especially those related to traction) and vehicle speed.

The adhesion coefficient is the measure commonly used for the characterization of wheel-rail adhesion. The estimation of wheel-rail adhesion coefficient is made on experimental basis, either using laboratory test rigs [8,9] or real field determinations, made for different track conditions, axle loads and running speeds. Based on the experimental data are derived empirical expressions of the wheel-rail adhesion coefficient. For example, in [8] is indicated the following expression of adhesion coefficient:

$$
\mu_{a}=0.005+\frac{13}{0.31 V+42},
$$

The formula being the result of experiments carried out on a full-scale roller rig, on which were simulated speeds of up to $280 \mathrm{~km} / \mathrm{h}$. Another expression, used in Japan for Shinkansen trains under wet conditions [10] is given by

$$
\mu_{a}=\frac{13.6}{V+85} \text {. }
$$

In [11] are presented expressions of the adhesion coefficient for dry and wet conditions:

$$
\mu_{a}=0.0624+\frac{45.6}{V+260} ; \mu_{a}=0.0405+\frac{13.5}{V+120},
$$

While in [12] is indicated a general formula

$$
\mu_{a}=\frac{\mu_{0}}{1+0.01 V}
$$

In the equations above $V$ is the train speed in $\mathrm{km} / \mathrm{h}$ and $\mu_{0}$ - in equation (4) - is a value of the adhesion coefficient that depends mainly on the conditions of the track (rail).

\section{Limitation of maximum speed of train by wheel-rail adhesion}

The equation of motion of a train is of the form:

$$
F_{T}-R_{T}=m(1+\gamma) a
$$

Where $F_{T}$ is the traction force of the train, provided by the motor vehicle(s); $m, a$ and $R_{T}$ are the train mass, acceleration and resistance force, respectively and $\gamma$ is a mass factor 
modelling the effect of accelerating rotational components of the vehicles. The total train resistance can be written as a sum of main resistance and additional resistances:

$$
R_{T}=R_{R}+R_{G}+R_{C}
$$

The main resistance $R_{R}$ is the one that act permanently on the train, namely the running resistance on straight and level track. The additional resistances are gradient resistance $R_{G}$ and curve resistance $R_{C}$, which occur only when the train travels on a slope or in curve, respectively. The running resistance of train $R_{R}$ includes the mechanical (rolling) resistance and the air resistance and it is usually expressed by an equation of the form $[1,4]$

$$
R_{R}=A+B V+C V^{2}
$$

Known as the "Davis equation", where $V$ is the train speed and $A, B, C$ are coefficients that depend on train (vehicle) characteristics: mass, bogie type, cross-section, length, shape, etc. The running resistance can be expressed as a specific resistance by dividing the absolute resistance in equation (7) to the train (vehicle) weight $G_{T}$ :

$$
r_{R}=\frac{R_{R}}{G_{T}}=a+b V+c V^{2} .
$$

When the train is running on a slope and in curve, the corresponding specific resistances must be added, so the total specific resistance is given by:

$$
r_{T}=r_{R}+r_{C} \pm i
$$

Where $r_{C}$ is the specific curving resistance and $i$ is the track gradient expressed in $\mathrm{mm} / \mathrm{m}$. The train total resistance force (expressed in N) can be obtained by multiplying the specific resistance in equation (9) by the weight of train (expressed in $\mathrm{kN}$ ):

$$
R_{T}=r_{T} G_{T} .
$$

Since the railway vehicles are of various shapes, sizes and design, the running resistance given by equation (8), i.e. the values of coefficients $a, b, c$, must be customized for each type of vehicle.

As it can be seen from equation (5), the train total traction force must overcome the resistance and inertia forces. The traction force is limited by wheel-rail adhesion force $F_{a}$ :

$$
F_{a}=\mu_{a} G_{a}
$$

Where $G_{a}$ is the adhesive weight, i.e. the total weight supported by driving axles of the train. For a driving axle, if the traction force is larger than the wheel-rail adhesion force, the wheelset slip occurs. The traction force of a motor vehicle is therefore limited by its adhesive weight and by the adhesion coefficient. Since the train total traction force cannot exceed the adhesion force given by equation (11), this latter one can therefore be considered as the upper limit of the traction force.

As it was shown above, when the train traveling speed is increasing, the running resistance is increasing, while the wheel-rail adhesion is decreasing. To increase train speed, the acceleration in relation (5) must be positive, so the traction force must be greater than the train total resistance. The train maximum theoretical speed is obtained when the total resistance reaches the adhesion force (regarded as the maximum usable traction force): 


$$
R_{T}\left(V_{\max }\right)=F_{a}\left(V_{\max }\right)
$$

\section{Case study}

In this section, a numerical application is carried out, based on the empirical formulas of wheel-rail adhesion coefficient and of train resistance presented in the previous sections. The analysis is performed considering usual operation conditions of high-speed trains, regarding the adhesion coefficient values [1,5,6,9] and the gradient superior limits [2]. Also, there are used the real data of two Japanese high-speed trains: the Shinkansen 700 and N700 series.

The wheel-adhesion coefficient is estimated using equation (4), the coefficient $\mu_{0}$ being considered within an interval of values, corresponding to the diverse conditions of the track (rail). It is to be noted that there are not considered the lowest possible values for $\mu_{0}-$ of 0.05 or even less - corresponding to the situation of contaminated surfaces with oil, wet dead leaves, etc. The reason for this is that such situations are occasional and intermittent and occur usually on short sections of track, thus affecting only on short term the wheel-rail adhesion. On the other hand, the situation of surfaces that are slightly wet (light rain, snow shower, dew) is quite common and may affect adhesion on extensive sections of track. In such a situation, the adhesion coefficient may drop to a value of 0.10 . Given all this, the present analysis is done considering values of the coefficient $\mu_{0}$ in a range between 0.12 and 0.32 , the lower value corresponding to slightly wet surfaces and the higher one to the ideal situation of dry and clean surfaces.

Regarding the track gradient $i$ which affects the total specific resistance of the train - see equation (9), its maximum value is considered $30 \mathrm{~mm} / \mathrm{m}$, an accepted slope for high-speed lines, as indicated in [2]. The curving resistance is not taken into account in the present study, given its reduced importance (high-speed lines have large curve radius).

The 700 series and $N 700$ ('New' 700) series are Japanese high-speed trains in service since 1998 and 2007, respectively. The basic operating formula for both trains is 16-car sets: 12 motor cars and 4 trailer cars for 700 series, 14 motor cars and 2 trailer cars for N700 series. Other trains data, relevant for the present analysis, are presented in Table 1 [1].

Table 1. Shinkansen trains data.

\begin{tabular}{|c|c|c|}
\hline & 700 series & N700 series \\
\hline Maximum operation speed $[\mathrm{km} / \mathrm{h}]$ & 285 & 300 \\
\hline Total mass $[\mathrm{t}]$ & 708 & 715 \\
\hline Adhesive mass $[\mathrm{t}]$ & 540 & 630 \\
\hline$a[\mathrm{~N} / \mathrm{kN}]$ & 1.127 & 0.836 \\
\hline$b[\mathrm{~N} /(\mathrm{kN} \cdot \mathrm{km} / \mathrm{h})]$ & $3.962 \cdot 10^{-3}$ & $8.721 \cdot 10^{-3}$ \\
\hline$c\left[\mathrm{~N} /\left(\mathrm{kN} \cdot \mathrm{km}^{2} / \mathrm{h}^{2}\right)\right]$ & $1.133 \cdot 10^{-4}$ & $7.857 \cdot 10^{-5}$ \\
\hline
\end{tabular}

Based on the above presented data and considering value ranges of the adhesion coefficient and track gradient, a numerical analysis is performed to obtain the maximum speed which meet the condition in equation (12). In Figure 1 are shown the results of this analysis for the two considered trains. It can be seen that a lower wheel-rail adhesion (lower values of coefficient $\mu_{0}$ ) results in a reduction of the maximum speed limited by adhesion. Also, a higher total train resistance - because of an increased gradient $i$ - entails a lower maximum speed limited by adhesion. A comparison between the results obtained for the two trains shows higher maximum speeds for the $N 700$ series, which is the newer and more technologically advanced train. The reasons for this are its lower specific resistance and its higher percentage of driving axles (14 motor cars out of 16 compared to only 12 out of 16 for the 700 series). 

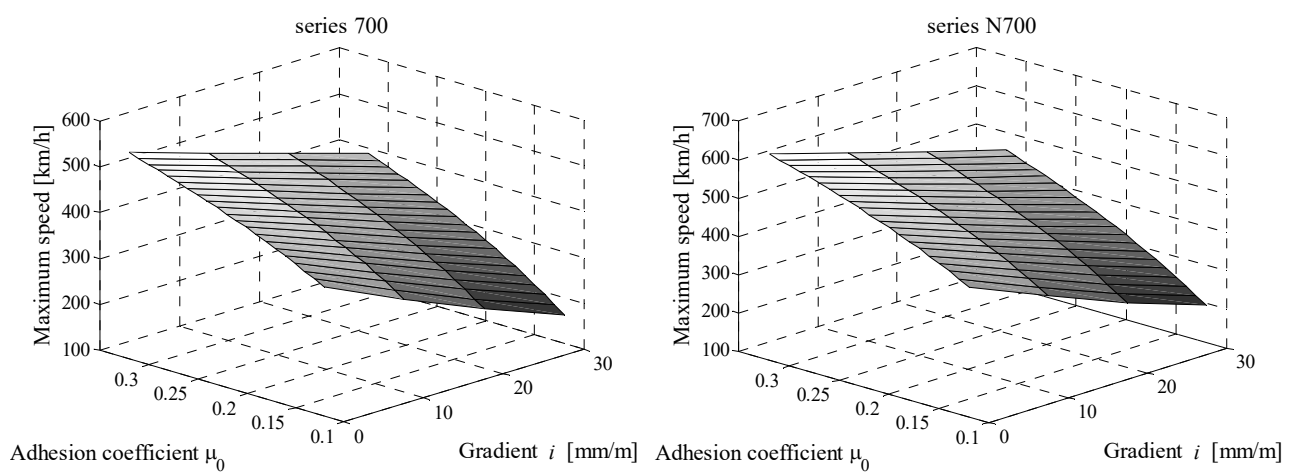

Fig. 1. Maximum speed limited by wheel-rail adhesion: series 700 (left) and N700 (right).

The results show that for reasonably high values of the adhesion coefficient $\mu_{0}$ or for low gradients both trains can reach the maximum operation speed. However, in the case of low adhesion and large gradients this condition is no longer fulfilled. In the worst-case scenario - the lowest adhesion and the higher gradient - the maximum speed is limited to $197 \mathrm{~km} / \mathrm{h}$ for the $N 700$ series train and to $163 \mathrm{~km} / \mathrm{h}$ for the 700 -series train.

An important issue is to evaluate what level of wheel-rail adhesion is required to allow the train to travel at the maximum operation speed in any circumstances. In Figure 2 can be seen the adhesion coefficient $\mu_{0}$ necessary so as the trains reach the maximum operation speed for different values of track gradient.

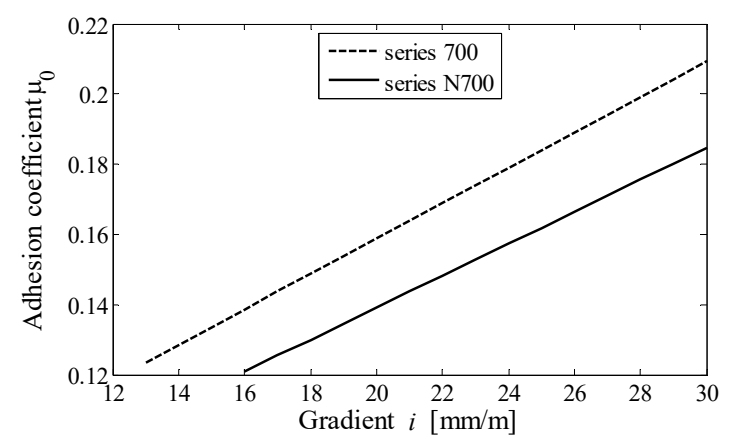

Fig. 2. Adhesion coefficient required to reach maximum operation speed.

For values higher than 0.21 of the adhesion coefficient $\mu_{0}$, both trains can reach the maximum operation speed in any conditions (i.e. even for the highest gradient). Also, for gradients lower than $12 \mathrm{~mm} / \mathrm{m}$, the maximum speed can be achieved even in the circumstances of the poorest adhesion $\left(\mu_{0}=0.12\right)$. The graph in Figure 2 confirms once again the superiority of the train series N700: although its maximum operation speed is higher than that of the 700 -series train $(300 \mathrm{~km} / \mathrm{h}$ versus $285 \mathrm{~km} / \mathrm{h})$, the $N 700$ train requires lower levels of adhesion to achieve its top operation speed. For example, in the case of a gradient of $30 \mathrm{~mm} / \mathrm{m}$, the $N 700$ train requires a value $\mu_{0}=0.185$ to reach $300 \mathrm{~km} / \mathrm{h}$, while the 700 train needs at least a value $\mu_{0}=0.21$ for achieving a speed of $285 \mathrm{~km} / \mathrm{h}$.

\section{Conclusion}

The wheel-rail adhesion is one of the most important issues in rail transport, affecting both traction and braking of trains. In the high-speed domain, adhesion is a limiting factor of the maximum speed of trains, because the maximum usable traction force is limited by the 
adhesion force, while the train resistance force, which must be overcome by the traction force, is increasing with speed.

An accurate evaluation of the maximum speed of trains limited by adhesion is hard to do, because it requires a precise evaluation of wheel-rail adhesion and train resistance. Due to the complexity of the phenomena involved and to the influence of many factors (most of them random), both adhesion coefficient and train resistance can be evaluated only on the basis on empirical formulas.

In the present paper, an analysis has been performed based on empirical formulas of wheel-rail adhesion and train resistances. There have been considered ranges of usual values for the track gradient and the adhesion coefficient and there have been used real data of two Japanese high-speed trains. The results confirmed that wheel-rail adhesion is an important factor in terms of train speed limitation. Low adhesion and high train resistance (high track gradient) resulted in limitation of train maximum speed, to levels significantly lower than the maximum operation one. This maximum operation speed can be reached for reasonably high levels of the adhesion or for low gradients; but, as the gradient increases, higher levels of adhesion are required.

Better performance has been achieved for the $N 700$ series train because of its lower specific resistance and its higher percentage of driving axles. This observation indicates, in fact, the ways through which train performance can be improved concerning the wheel-rail adhesion issue. To increase the maximum speed limited by adhesion, the adhesion force must be increased and the resistance force must be reduced. Since the wheel-rail adhesion and the resistances given by track gradient and curve are practically impossible to influence, measures should focus on increasing the adherent weight (by increasing the percentage of driving axles of the train) and on reducing train running resistance, especially the aerodynamic drag, which is predominant at high speeds.

\section{References}

1. M. Spiroiu, High-speed technique in rail transport (in Romanian) (Matrix Rom, Bucharest, 2012)

2. High Speed Rail - Fast Track to Sustainable Mobility (UIC, Paris, 2015)

3. M.A. Spiroiu, IOP Conf. Series: Mater. Sci. Eng., 147, 012117 (2016)

4. S. Iwnicki, Handbook of Railway Vehicle Dynamics (CRC Press, Boca Raton, 2006)

5. W. Wang, H. Zhang, H. Wang, Q. Liu, M. Zhu, Wear, 271 (9-10), 2693-2698 (2011)

6. B. Allotta, E. Melin, A. Ridolfi, A. Rindi, Tribol. Int., 69, 128-140 (2014)

7. G. Trummer, L.E. Buckley-Johnstone, P. Voltr et al., Tribol. Int., 109, 409-415 (2017)

8. W. Zhang, J. Chen, X. Wu, X. Jin, Wear, 253 (1-2), 82-88 (2002)

9. W.J. Wang, H. Wang, H.Y. Wang, J. Guo et al., Wear, 302 (1-2), 1461-1467 (2013)

10. A. Mochizuki, Japan Railway \& Transp. Rev., 57, 42-49 (2011)

11. W. Haijun, J. Zeng, R. Luo, Wear, 314 (1-2), 299-304 (2014)

12. J.A Lozano., J. Félez, J. de Dios Sanz, J. M. Mera, Railway Traction In: Reliability and Safety in Railway (InTech, 2012). Available from: https://www.intechopen.com/books/reliability-and-safety-in-railway/railway-traction 